\title{
Glicerina semipurificada vegetal e mista na alimentação de coelhos em crescimento
}

\author{
[Vegetal and mixed semipurified glycerin in the feeding of growing rabbits] \\ M. Retore ${ }^{1}$, C. Scapinello ${ }^{2}$, I. Moreira ${ }^{2}$, I.G. Araujo ${ }^{2}$, B. Ponciano Neto ${ }^{2}$, \\ C.E. Stanquevis ${ }^{2}$, A.F.G. Oliveira ${ }^{2}$ \\ ${ }^{1}$ Embrapa Agropecuária Oeste - Dourados, MS \\ ${ }^{2}$ Universidade Estadual de Maringá - Maringá, PR
}

\begin{abstract}
RESUMO
Dois experimentos foram conduzidos com o objetivo de determinar a energia digestível da glicerina semipurificada vegetal e mista e o desempenho de coelhos em crescimento alimentados com dietas contendo os coprodutos. No ensaio de digestibilidade, foram utilizados 108 coelhos da raça Nova Zelândia Branco, com 45 dias de idade, distribuídos ao acaso em nove tratamentos $(0,4,8,12$ e $16 \%$ de inclusão de glicerina vegetal e mista na dieta), com 12 repetições. Para o ensaio de desempenho, utilizaram-se 180 coelhos da raça Nova Zelândia Branco, dos 32 aos 70 dias de idade, distribuídos ao acaso em arranjo fatorial $2 \times 4$ (dois tipos de glicerina e quatro porcentagens de inclusão: 3, 6, 9 e 12\%) mais uma dieta referência, com 10 repetições e dois animais por unidade experimental. Glicerinas vegetal e mista apresentaram energia digestível de 4.048 e $3.697 \mathrm{kcal} / \mathrm{kg}$ MS, respectivamente. Entre 32 e 50 dias de idade dos coelhos, a dieta com $12 \%$ de glicerina mista prejudicou $(\mathrm{P}<0,05)$ o ganho de peso $(32,14$ $\mathrm{g} / \mathrm{dia})$, a conversão alimentar $(3,57)$ e o custo por quilo de ganho de peso $(\mathrm{R} \$ 2,08)$ em relação à dieta referência (39,42g/dia, 2,87 e $R \$ 1,69$, respectivamente). Dos 32 aos 70 dias, o ganho de peso (30,11 g/dia) e a conversão alimentar $(3,99)$ dos animais que receberam $12 \%$ de glicerina mista na dieta foram piores $(\mathrm{P}<0,05)$ em relação aos da dieta referência $(34,00 \mathrm{~g} / \mathrm{dia}$ e 3,65 , respectivamente). Para as características de desempenho, dos 32 aos 70 dias, não foram verificadas diferenças $(\mathrm{P}>0,05)$ entre a inclusão de glicerina vegetal na dieta e a dieta referência, porém a maior viabilidade econômica $(\mathrm{P}<0,05)$ foi com $12 \%$ de inclusão ( $\mathrm{R} \$ 1,89 \times \mathrm{R} \$ 2,15$, respectivamente). A glicerina vegetal pode ser incluída em até $12 \%$ da dieta e a mista, em até $9 \%$, diminuindo o custo de produção sem afetar o desempenho animal.
\end{abstract}

Palavras-chave: coelho, conversão alimentar, custo, ganho de peso, transesterificação catalítica

\begin{abstract}
Two trials were conducted with the goal of determining the digestible energy of vegetal and mixed semi purified glycerin and the performance of growing rabbits fed with diets containing the byproducts. In the digestibility assay 108 New Zealand White rabbits, 45 days old, were used, assigned in a completely randomized design into nine treatments $(0,4,8,12$ and $16 \%$ of glycerins inclusion), with 12 replications. The treatment with $0 \%$ of glycerin inclusion was considered the reference diet. In the performance assay, 180 New Zealand White rabbits, 32 to 70 days of age, were assigned to a completely randomized design into a $2 \times 4$ factorial arrangement (two types of glycerin and four percentages of inclusion: 3, 6, 9 and $12 \%)$ plus a reference diet and ten replications with two animals per experimental unit. Vegetal and mixed glycerin showed a digestible energy of 4,048 and 3,697 kcal/kg of dry matter, respectively. From 32 to 50 days of age, the diet with $12 \%$ of mixed glycerin affected $(P<0.05)$ weight gain (32.14g/day), feed conversion (3.57) and cost per kilo of weight gain $(R \$ 2.08)$ in relation to the reference diet (39.42g/day, 2.87 and $R \$ 1.69$, respectively). From 32 to 70 days, weight gain (30.11g/day) and feed conversion (3.99) of the animals fed with the diet containing $12 \%$ of mixed glycerin were worst $(P<0.05)$ in relation to the reference diet (34.00 g/day and 3.65, respectively). Regarding characteristics of animal
\end{abstract}

Recebido em 9 de junho de 2011

Aceito em 10 de setembro de 2012

E-mail: marciana@cpao.embrapa.br 
performance, from 32 to 70 days, no differences $(P>0.05)$ were observed between the inclusion of vegetal glycerin in the diet and the reference diet, but better economic viability was verified $(P<0.05)$ with the diet containing $12 \%$ of vegetal glycerin ( $R \$ 1.89 \times R \$ 2.15$, respectively). Vegetal glycerin can be included up to $12 \%$ in the diet and mixed up to $9 \%$, reducing production cost without affecting animal performance.

Keywords: catalytic transesterification, cost, feed conversion, rabbit, weight gain

\section{INTRODUÇÃO}

Os níveis elevados de amido na dieta de coelhos recém-desmamados podem causar disfunções digestivas graves, as quais podem levar o animal à morte, visto que a atividade enzimática específica para a sua digestão, a amilase, ainda está imatura (Gidenne, 1997). Na tentativa de reduzir a quantidade desse ingrediente na dieta de coelhos jovens, óleos ou gorduras são adicionados. Entretanto, os níveis de inclusão restringem-se ao máximo de $4 \%$ da dieta, por razões de custo e/ou qualidade de pélete. Assim, alimentos alternativos ao uso dessas matériasprimas na ração que possam melhorar o desempenho animal, sem causar transtornos digestivos, e ainda diminuir o custo de produção precisam ser mais bem estudados. Um deles é a glicerina.

A glicerina é obtida durante o processo de produção do biodiesel por reação de transesterificação catalítica dos triacilgliceróis de diferentes oleaginosas ou gordura animal com álcoois (etanol ou metanol). É composta por glicerol, resíduos de catalisadores, sabão, álcool, monoacilglicerol, diacilglicerol, oligômeros de glicerol e água (Ooi et al., 2004) e apresenta-se na forma de líquido viscoso pardo escuro.

As glicerinas diferenciam-se pelo grau do processamento industrial, o qual pode ser tanto na forma bruta (alto conteúdo de ácidos graxos) quanto na forma semipurificada (baixo conteúdo de ácidos graxos). Podem ser produzidas através de fontes vegetais, animais ou pela associação de ambas (mista). Para obtenção da glicerina pura (acima de 99\% de glicerol), é necessário processo de destilação e descoloração (Yong et al., 2001), o que eleva muito seu custo. Assim, predomina no mercado nacional a oferta de glicerina bruta ou de baixa pureza (50 a 70\% de glicerol) e glicerina semipurificada ou de média pureza (80 a $90 \%$ de glicerol), conforme classificação de Südekum (2008).

Assim, o presente trabalho teve por objetivos determinar a energia digestível das glicerinas semipurificadas vegetal e mista e avaliar diferentes porcentagens de inclusão dos coprodutos na dieta sobre o desempenho, peso de carcaça e rendimento das vísceras comestíveis de coelhos em crescimento.

\section{MATERIAL E MÉTODOS}

Dois ensaios experimentais foram realizados no setor de cunicultura da fazenda experimental de Iguatemi; um com intuito de determinar a energia digestível das glicerinas e outro para avaliar o desempenho dos animais alimentados com diferentes porcentagens de inclusão dos coprodutos na dieta. $\mathrm{O}$ ensaio de digestibilidade foi conduzido no mês de abril e o de desempenho nos meses de outubro e novembro de 2009.

Foram estudadas duas glicerinas semipurificadas: glicerina semipurificada vegetal, oriunda exclusivamente de óleo vegetal (soja) e glicerina semipurificada mista, oriunda da mistura de óleo vegetal com gordura animal. As glicerinas utilizadas foram fornecidas pela indústria de biocombustíveis Biopar, situada em Rolândia, PR, e suas composições químicas são apresentadas na Tab. 1.

Para o ensaio de digestibilidade foram utilizados 108 coelhos, metade machos e metade fêmeas, com 45 dias de idade, da raça Nova Zelândia Branco. Os animais foram alojados individualmente em gaiolas de metabolismo, providas de bebedouro automático tipo chupeta, comedouro semiautomático de chapa galvanizada e dispositivo para coleta de fezes. 
Glicerina semipurificada...

Tabela 1. Composição química da glicerina semipurificada vegetal e mista, com base na matéria seca

\begin{tabular}{lcc}
\hline Variável & $\begin{array}{c}\text { Glicerina semipurificada } \\
\text { vegetal }\end{array}$ & $\begin{array}{c}\text { Glicerina semipurificada mista } \\
\text { Matéria seca, \% }\end{array}$ 95,62 \\
Cinzas, \% & 2,15 & 85,68 \\
Ácidos graxos totais, \% & 9,00 & 3,76 \\
Glicerol, \% & 78,30 & 5,10 \\
Energia bruta, kcal/kg & 4.089 & 68,66 \\
Sódio, \% & 0,91 & 3.751 \\
Cloro, \% & 0,38 & 1,21 \\
Potássio, \% & 0,12 & 0,44 \\
Cálcio, \% & 0,03 & 0,01 \\
Fósforo, \% & 0,16 & 0,09 \\
Proteína bruta, \% & 0,06 & 0,76 \\
\hline
\end{tabular}

O delineamento utilizado foi o inteiramente ao acaso com nove tratamentos, sendo uma dieta referência e oito dietas testes em que as glicerinas semipurificadas vegetal e mista foram incluídas em 4, 8, 12 e 16\% nas dietas testes, com 12 repetições. A dieta referência foi composta por milho moído, feno de capimestrela, feno de alfafa, farelo de trigo, farelo de soja, calcário calcítico, fosfato bicálcico, suplemento mineral+vitamínico, DL-Metionina, L-Lisina $\mathrm{HCl}$ e coccidiostático. Com essa formulação obtiveram-se $17 \%$ de proteína bruta, $2.450 \mathrm{kcal} / \mathrm{kg}$ de energia digestível, $44 \%$ de fibra em detergente neutro, $22 \%$ de fibra em detergente ácido e $21 \%$ amido.

Com base na matéria natural da dieta referência (DR), foram feitas as inclusões das glicerinas $(96 \% \mathrm{DR}+4 \%$ glicerina, $92 \% \mathrm{DR}+8 \%$ glicerina, $88 \%$ DR $+12 \%$ glicerina e $84 \%$ DR + $16 \%$ glicerina), obtendo-se oito dietas testes. O ensaio teve duração de 14 dias, sendo 10 dias para adaptação às gaiolas e às dietas e quatro dias para a coleta de fezes, segundo o Método de Referência Europeu para experimento de digestibilidade in vivo (Perez et al., 1995). Durante todo o experimento, os animais foram alimentados à vontade com livre acesso à água.

As fezes de cada animal foram coletadas, na sua totalidade, uma vez ao dia, no período da manhã, acondicionadas em sacos plásticos e armazenadas em freezer à temperatura de $-10^{\circ} \mathrm{C}$. Posteriormente, foram colocadas em estufa de ventilação forçada a $55^{\circ} \mathrm{C}$, durante $72 \mathrm{~h}$. Após homogeneização, parte da amostra foi moída em moinho com peneira de 1,0 $\mathrm{mm}$ para as análises de matéria seca, de acordo com Silva e Queiroz (2002). Os valores de energia bruta foram determinados por meio de calorímetro adiabático (Parr Instrument Co. AC720), segundo os procedimentos descritos por Silva e Queiroz (2002). Os coeficientes de digestibilidade da matéria seca e da energia bruta da glicerina vegetal e mista foram calculados conforme Matterson et al. (1965). Os valores da matéria seca digestível e da energia digestível foram estimados pela análise de regressão de Adeola e Ileleji (2009).

No experimento de desempenho foram utilizados 180 coelhos da raça Nova Zelândia Branco, machos e fêmeas, no período de 32 a 70 dias de idade, alojados em gaiolas de arame galvanizado. A temperatura média mínima no galpão foi de $19,7^{\circ} \mathrm{C} \pm 2,15$ e a média máxima, de $25,9^{\circ} \mathrm{C} \pm 3,58$.

Os animais foram distribuídos em delineamento inteiramente ao acaso com nove tratamentos e 10 repetições, com dois animais, um macho e uma fêmea, por unidade experimental. Os tratamentos consistiram em uma dieta referência, formulada de acordo com as exigências para coelhos em crescimento (Blas e Wiseman, 1998) e outras oito dietas, contendo 3, 6, 9 e $12 \%$ de glicerina semipurificada vegetal e mista (Tab. 2). As dietas experimentais foram peletizadas a seco e, durante todo o experimento, os animais foram alimentados à vontade com livre acesso à água. 
Tabela 2. Composição percentual e química das dietas experimentais para coelhos, dos 32 aos 70 dias de idade

\begin{tabular}{|c|c|c|c|c|c|c|c|c|c|}
\hline \multirow{2}{*}{ Ingrediente } & \multirow{2}{*}{ DR } & \multicolumn{4}{|c|}{ Semipurificada vegetal (\%) } & \multicolumn{4}{|c|}{ Semipurificada mista (\%) } \\
\hline & & 3 & 6 & 9 & 12 & 3 & 6 & 9 & 12 \\
\hline Milho moído & 22,10 & 19,08 & 16,05 & 13,01 & 10,00 & 18,84 & 15,55 & 12,27 & 9,00 \\
\hline Glicerina & 0,00 & 3,00 & 6,00 & 9,00 & 12,00 & 3,00 & 6,00 & 9,00 & 12,00 \\
\hline $\begin{array}{l}\text { Feno capim- } \\
\text { estrela }\end{array}$ & 22,60 & 22,65 & 22,70 & 22,75 & 22,80 & 21,80 & 21,00 & 20,20 & 19,40 \\
\hline Feno de alfafa & 15,00 & 15,50 & 16,00 & 16,50 & 17,00 & 15,50 & 16,00 & 16,50 & 17,00 \\
\hline Farelo de trigo & 23,00 & 22,00 & 21,00 & 20,00 & 19,00 & 23,00 & 23,00 & 23,00 & 23,00 \\
\hline Farelo de soja & 13,00 & 13,75 & 14,50 & 15,25 & 16,00 & 13,62 & 14,25 & 14,87 & 15,50 \\
\hline Calcário calcítico & 0,80 & 0,75 & 0,70 & 0,65 & 0,60 & 0,76 & 0,72 & 0,68 & 0,64 \\
\hline Fosfato bicálcico & 0,40 & 0,46 & 0,52 & 0,58 & 0,64 & 0,42 & 0,45 & 0,47 & 0,50 \\
\hline Sal comum & 0,40 & 0,37 & 0,35 & 0,33 & 0,30 & 0,37 & 0,35 & 0,33 & 0,30 \\
\hline Suplemento & 0,50 & 0,50 & 0,50 & 0,50 & 0,50 & 0,50 & 0,50 & 0,50 & 0,50 \\
\hline $\mathrm{Min}+\mathrm{Vit}^{1}$ & & & & & & & & & \\
\hline DL-Metionina & 0,09 & 0,09 & 0,09 & 0,10 & 0,10 & 0,09 & 0,09 & 0,10 & 0,10 \\
\hline L-Lisina $\mathrm{HCl}$ & 0,05 & 0,04 & 0,03 & 0,02 & 0,00 & 0,04 & 0,03 & 0,02 & 0,00 \\
\hline Coccidiostático $^{2}$ & 0,06 & 0,06 & 0,06 & 0,06 & 0,06 & 0,06 & 0,06 & 0,06 & 0,06 \\
\hline Óleo de soja & 2,00 & 1,75 & 1,50 & 1,25 & 1,00 & 2,00 & 2,00 & 2,00 & 2,00 \\
\hline Nutriente (\%) & \multicolumn{9}{|c|}{ Composição, base na \% MS } \\
\hline $\mathrm{Amido}^{3}$ & 21,10 & 19,00 & 16,90 & 15,70 & 14,50 & 19,10 & 18,40 & 16,25 & 14,10 \\
\hline $\mathrm{ED}, \mathrm{kcal} / \mathrm{kg}$ & 2.650 & 2.650 & 2.650 & 2.650 & 2.650 & 2.650 & 2.650 & 2.650 & 2.650 \\
\hline $\mathrm{FDN}^{4}$ & 42,00 & 42,50 & 43,00 & 42,50 & 42,00 & 42,00 & 42,00 & 40,60 & 39,15 \\
\hline $\mathrm{FDA}^{4}$ & 20,00 & 20,70 & 21,40 & 21,80 & 22,15 & 21,00 & 21,90 & 21,25 & 20,60 \\
\hline PB & 16,00 & 16,00 & 16,00 & 16,00 & 16,00 & 16,00 & 16,00 & 16,00 & 16,00 \\
\hline Cálcio & 0,80 & 0,80 & 0,80 & 0,80 & 0,80 & 0,80 & 0,80 & 0,80 & 0,80 \\
\hline Fósforo & 0,50 & 0,50 & 0,50 & 0,50 & 0,50 & 0,50 & 0,50 & 0,50 & 0,50 \\
\hline Lisina & 0,76 & 0,76 & 0,76 & 0,76 & 0,76 & 0,76 & 0,76 & 0,76 & 0,76 \\
\hline Met+Cis & 0,60 & 0,60 & 0,60 & 0,60 & 0,60 & 0,60 & 0,60 & 0,60 & 0,60 \\
\hline $\mathrm{EE}^{4}$ & 4,50 & 4,40 & 4,30 & 3,70 & 3,10 & 4,40 & 4,30 & 5,05 & 5,80 \\
\hline Custo/kg (R\$) & 0,59 & 0,58 & 0,57 & 0,57 & 0,56 & 0,58 & 0,58 & 0,58 & 0,58 \\
\hline
\end{tabular}

DR: dieta referência; ED: energia digestível; FDN: fibra em detergente neutro; FDA: fibra em detergente ácido; PB:

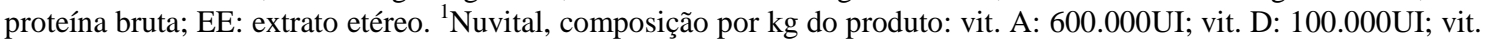
E: $8.000 \mathrm{mg}$; vit. K3: 200mg; vit. B1: 400mg; vit. B2: 600mg; vit. B6: 200mg; vit. B12: 2.000 mcg; ácido pantotênico: 2.000mg; colina: 70.000mg; Fe: $8.000 \mathrm{mg}$; Cu: $1.200 \mathrm{mg}$; Co: 200mg; Mn: $8.600 \mathrm{mg}$; Zn: $12.000 \mathrm{mg}$; I: 64mg; Se: $16 \mathrm{mg}$; Metionina: $120.000 \mathrm{mg}$; antioxidante: $20.000 \mathrm{mg} .{ }^{2}$ Princípio ativo à base de robenidina $(6,6 \%)$. ${ }^{3}$ Análise realizada pelo Instituto de Tecnologia de Alimentos, de Campinas. ${ }^{4}$ Análises realizadas no Laboratório de Alimentos e Nutrição Animal do Departamento de Zootecnia da UEM.

As dietas e os animais foram pesados no início do experimento (aos 32 dias de idade), aos 50 dias e no final do experimento (aos 70 dias de idade). As características de desempenho avaliadas foram o peso vivo, ganho de peso diário, consumo de ração diário, conversão alimentar e custo por quilo de peso vivo ganho. $\mathrm{O}$ abate dos animais foi realizado sem jejum prévio, com atordoamento e posterior corte da jugular. Para a determinação do peso da carcaça, considerou-se a carcaça quente com a cabeça e sem vísceras comestíveis (coração, fígado e rins). Foram obtidos também os rendimentos de coração, fígado e rins.
Para verificar a viabilidade econômica da inclusão das glicerinas na dieta, utilizou-se a equação descrita por Bellaver et al. (1985), que calcula o custo médio da ração por quilograma de peso vivo ganho. Para calcular os custos das dietas experimentais, foram utilizados os preços dos insumos da região de Maringá - PR durante o mês de julho de 2010. Os resultados das variáveis estudadas no ensaio de desempenho foram analisados pelo programa estatístico SAEG (Sistema..., 2007), de acordo com o seguinte modelo estatístico: $\mathrm{Y}_{\mathrm{ijk}}=\mathrm{b}_{0}+\mathrm{Ti}+\mathrm{b}_{1 \mathrm{i}}$ $(\mathrm{Nij}-\tilde{\mathrm{Ni}})+\mathrm{b}_{2 \mathrm{i}}(\mathrm{Nij}-\tilde{\mathrm{Ni}})+\mathrm{FA}+\mathrm{e}_{\mathrm{ijk}}$, em que: $\mathrm{Y}_{\mathrm{ijk}}=$ variável medida na unidade experimental 
$\mathrm{k}$, alimentada com o tipo de dieta i, com inclusão $\mathrm{j}$ de glicerina semipurificada vegetal ou mista; $\mathrm{b}_{0}$ = constante geral; $\mathrm{Ti}=$ efeito do tipo de dieta $\mathrm{i}$, sendo $\mathrm{i} 1=$ dieta referência, $\mathrm{i} 2=$ dietas contendo glicerina semipurificada vegetal e i3 = dietas contendo glicerina semipurificada mista; $b_{1 \mathrm{i}}=$ coeficiente de regressão linear em função da inclusão $\mathrm{j}$ de glicerina na dieta do tipo $\mathrm{i}$, para todo $\mathrm{i}$ diferente de $1 ; \mathrm{b}_{2}=$ coeficiente de regressão quadrático em função da inclusão $\mathrm{j}$ de glicerina na dieta do tipo i, para todo i diferente de 1 ; Nij = inclusão $\mathrm{j}$ da glicerina adicionada no tipo de dieta i, para todo i diferente de $1 ; \tilde{\mathrm{N}} \mathrm{i}=$ porcentagem média da glicerina adicionada na dieta do tipo i, para todo i diferente de $1 ; \mathrm{FA}=$ falta de ajuste do modelo de regressão; $\mathrm{e}_{\mathrm{ij}}=$ erro aleatório associado a cada observação.

Para a comparação dos resultados obtidos com a dieta referência e aqueles obtidos com cada uma das inclusões de glicerina, foi aplicado o teste de Dunnett $(\mathrm{P}<0,05)$.

\section{RESULTADOS E DISCUSSÃO}

O milho apresenta energia bruta de $3.925 \mathrm{kcal} / \mathrm{kg}$ (Rostagno et al., 2005) e coeficiente de digestibilidade da energia para coelhos próximo a $100 \%$ (Blas e Gidenne, 1998). As glicerinas semipurificadas avaliadas no presente trabalho apresentam elevado coeficiente de digestibilidade e energia semelhante ao do cereal (Tab. 3), tornando-se matéria-prima em potencial na alimentação dessa espécie animal. Com $9 \%$ de inclusão de glicerina como ingrediente energético nas rações para coelhos jovens, é possível reduzir em torno de $24 \%$ a quantidade de amido presente na dieta, minimizando as chances de ocorrer algum distúrbio digestivo.

Lammers et al. (2008), ao trabalharem com glicerina com $86,95 \%$ de glicerol e energia bruta de $3.625 \mathrm{kcal} / \mathrm{kg}$ para suínos em crescimento, encontraram coeficientes de digestibilidade da energia entre 89 e $92 \%$, valores estes inferiores aos encontrados com coelhos no presente trabalho. Para frangos de corte, a digestibilidade da energia da glicerina tem ficado em torno de 75\% (Simon et al., 1996), também inferior aos valores encontrados no presente estudo. Diante desses resultados, é possível inferir que a espécie animal e a composição química da glicerina utilizada influenciam diretamente a digestibilidade da energia e o seu respectivo aproveitamento.

Tabela 3. Coeficientes de digestibilidade aparente (CD) e valores digestíveis dos dois tipos de glicerina semipurificada

\begin{tabular}{lcc} 
Coeficientes de digestibilidade, $\%$ & $\begin{array}{c}\text { Glicerina semipurificada } \\
\text { vegetal }\end{array}$ & $\begin{array}{c}\text { Glicerina semipurificada } \\
\text { mista }\end{array}$ \\
\hline CD da matéria seca & 96,81 & 98,68 \\
CD da energia bruta & 99,00 & 98,56 \\
\hline Nutrientes digestíveis & \multicolumn{2}{c}{ Matéria natural } \\
\hline Matéria seca digestível, \% & 92,57 & 84,55 \\
Energia digestível, $\mathrm{kcal} / \mathrm{kg}$ & 4.048 & 3.697 \\
\hline
\end{tabular}

Para obter a energia digestível de cada glicerina, foi estimada a inclinação da relação linear consumo de energia digestível versus consumo de glicerina vegetal e mista (Fig. 1), mostrando que a glicerina semipurificada vegetal apresentou maior quantidade de energia digestível $(4.048 \mathrm{kcal} / \mathrm{kg})$ em comparação à glicerina semipurificada mista $(3.697 \mathrm{kcal} / \mathrm{kg})$. Isso é justificado pela maior porcentagem de ácidos graxos presentes na glicerina vegetal (Tab. 1).
Analisando os resultados do experimento de desempenho, houve interação tipo de glicerina versus porcentagem de inclusão para as variáveis de peso vivo, ganho de peso diário, conversão alimentar e custo por $\mathrm{kg}$ de peso vivo ganho durante o período de 32 a 50 dias de idade (Tab. 4). Embora o consumo de ração tenha sido semelhante entre os tratamentos $(\mathrm{P}>0,05)$, foi verificado comportamento quadrático para todas as variáveis de desempenho dos animais que consumiram as dietas com inclusão de glicerina semipurificada mista, com ponto de máxima de 
$5,74 \%$ para ambos, peso vivo e ganho de peso diário, enquanto a conversão alimentar e custo por $\mathrm{kg}$ apresentaram ponto de mínima de $5,85 \%$ e 5,90\%, respectivamente. Aplicando-se o teste de Dunnett, apenas a dieta com a máxima inclusão de glicerina mista (12\%) apresentou resultados piores $(\mathrm{P}<0,05)$ em relação à dieta referência para as características acima mencionadas.

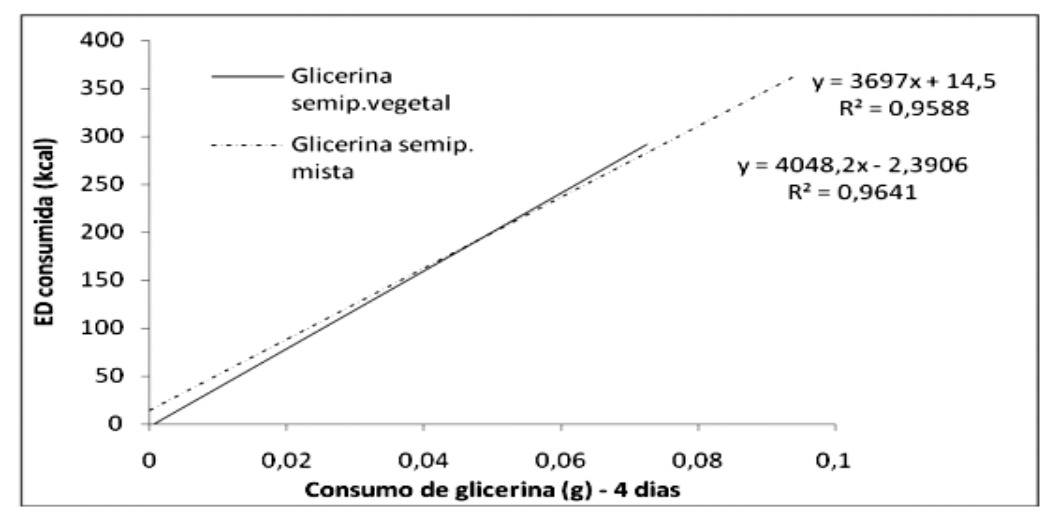

Figura 1. Estimativa da energia digestível das glicerinas semipurificadas

Tabela 4. Médias estimadas das características de desempenho e custo da ração por quilo de peso vivo ganho de coelhos alimentados, dos 32 aos 50 dias de idade, com dietas contendo glicerina semipurificada vegetal (GSV) e mista (GSM)

\begin{tabular}{|c|c|c|c|c|c|c|c|c|c|c|c|c|c|c|c|}
\hline \multirow{2}{*}{$\begin{array}{l}\text { Glicerina } \\
(\%)\end{array}$} & \multicolumn{3}{|c|}{ PV 50 (g) } & \multicolumn{3}{|c|}{ GPD $32-50(\mathrm{~g})$} & \multicolumn{3}{|c|}{ CRD 32-50 (g) } & \multicolumn{3}{|c|}{ CA $32-50$} & \multicolumn{3}{|c|}{ Custo/kg GP 32-50 } \\
\hline & $\overrightarrow{0}$ & 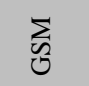 & 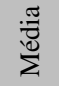 & $\vec{v}$ & 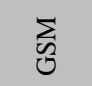 & 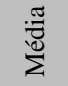 & $\overrightarrow{0}$ & 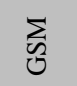 & 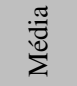 & $\overrightarrow{0}$ & 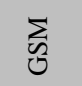 & 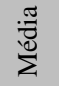 & $\overrightarrow{0}$ & 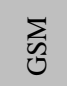 & 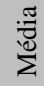 \\
\hline 3 & $1496 \mathrm{a}$ & $1519 \mathrm{a}$ & 1507 & $39,38 \mathrm{a}$ & $40,64 \mathrm{a}$ & 40,01 & 107,00 & 110,45 & 108,72 & $2,77 \mathrm{a}$ & $2,73 \mathrm{a}$ & 2,75 & $1,60 \mathrm{a}$ & $1,59 \mathrm{a}$ & 1,59 \\
\hline 6 & $1469 a$ & $1515 \mathrm{a}$ & 1492 & $37,89 a$ & $40,44 \mathrm{a}$ & 39,16 & 108,63 & 111,89 & 110,26 & $2,94 a$ & $2,88 \mathrm{a}$ & 2,91 & $1,68 \mathrm{a}$ & $1,66 \mathrm{a}$ & 1,67 \\
\hline 9 & $1452 b$ & $1523 a$ & 1487 & $36,93 b$ & $40,88 \mathrm{a}$ & 38,90 & 108,41 & 105,47 & 106,94 & $3,02 \mathrm{a}$ & $2,62 b$ & 2,82 & $1,72 \mathrm{a}$ & $1,52 \mathrm{~b}$ & 1,62 \\
\hline 12 & $1515 \mathrm{a}$ & $1366 * \mathrm{~b}$ & 1440 & $40,40 \mathrm{a}$ & $32,14 * \mathrm{~b}$ & 36,27 & 107,90 & 110,41 & 109,15 & $2,73 \mathrm{~b}$ & $3,57 * \mathrm{a}$ & 3,15 & $1,53 \mathrm{~b}$ & $2,08 \mathrm{a}$ & 1,80 \\
\hline Média & 1483 & 1481 & & 38,65 & 38,52 & & 107,98 & 109,55 & & 2,86 & 2,95 & & 1,63 & 1,71 & \\
\hline Regressão & $\mathrm{ns}$ & Quadr. & & $\mathrm{ns}$ & Quadr. & & & & $\mathrm{ns}$ & $\mathrm{ns}$ & Quadr. & & $\mathrm{ns}$ & Quadr. & \\
\hline
\end{tabular}

DR: dieta referência; PV50: peso vivo 50 dias; GPD 32-50: ganho de peso diário 32-50 dias; CRD 32-50: consumo de ração diário 32-50 dias; CA 32-50: conversão alimentar 32-50 dias; Custo/kg GP32-50: custo das rações, em reais, para cada quilo de ganho de peso dos 32 aos 50 dias de idade dos animais; CV: coeficiente de variação.

*Diferem da referência pelo teste de Dunnett $(\mathrm{P}<0,05)$.

Letras na linha representam o desdobramento da interação pelo teste Tukey $(\mathrm{P}<0,05)$. Ausência de letras indica que não houve interação.

ns= não significativo.

PV 50: $\mathrm{Y}=1400,62+49,5704 \mathrm{GSM}-4,3155 \mathrm{GSM}^{2} ; \mathrm{R}^{2}=0,33$

GPD 32-50: $\mathrm{Y}=34,0735+2,7539 \mathrm{GSM}-0,2397 \mathrm{GSM}^{2} ; \mathrm{R}^{2}=0,33$.

CA 32-50: $\mathrm{Y}=3,4056-0,2668 \mathrm{GSM}+0,0228 \mathrm{GSM}^{2} ; \mathrm{R}^{2}=0,30$.

Custo/kg GP: Y = 1,9954-0,1639GSM+0,0139GSM²; $\mathrm{R}^{2}=0,32$.

Sabe-se que o glicerol absorvido é metabolizado a glicerol-3-fosfato e aos intermediários da glicólise, dihidroxiacetona fosfato e gliceraldeído-3-fosfato. Assim, a possível causa para o pior desempenho pode ser explicada pelo menor aporte energético metabólico proporcionado pela adição de $12 \%$ de glicerina mista na dieta, por esta apresentar menor quantidade de ácidos graxos e glicerol do que a glicerina vegetal, uma vez que o período entre o desmame e os 50 dias de idade é o período em que os animais apresentam seu maior crescimento e, consequentemente, maior exigência nutricional. 
Para o período total de avaliação, dos 32 aos 70 dias de idade, foi observada interação $(\mathrm{P}<0,05)$ tipo de glicerina versus porcentagem de inclusão para peso vivo, ganho de peso diário, conversão alimentar e custo da ração por $\mathrm{kg}$ de peso vivo ganho. No entanto, não foi verificada diferença entre os tipos de glicerina estudados (Tab. 5).

Tabela 5. Médias estimadas das características de desempenho e custo da ração por quilo de peso vivo ganho de coelhos alimentados, dos 32 aos 70 dias de idade, com dietas contendo glicerina semipurificada vegetal (GSV) e mista (GSV)

\begin{tabular}{|c|c|c|c|c|c|c|c|c|c|c|c|c|c|c|c|}
\hline \multirow{2}{*}{$\begin{array}{l}\text { Glicerina } \\
(\%)\end{array}$} & \multicolumn{3}{|c|}{ PV $70(\mathrm{~g})$} & \multicolumn{3}{|c|}{ GPD 32-70 (g) } & \multicolumn{3}{|c|}{ CRD 32-70 (g) } & \multicolumn{3}{|c|}{ CA $32-70$} & \multicolumn{3}{|c|}{ Custo/kg GP 32-70 } \\
\hline & 3 & 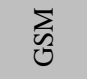 & 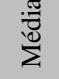 & $\vec{b}$ & 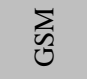 & 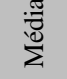 & $\vec{b}$ & 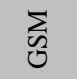 & 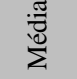 & $\overrightarrow{0}$ & $\sum_{\mathcal{U}}$ & 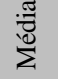 & $\vec{U}$ & $\sum_{\mathcal{U}}$ & 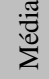 \\
\hline DR & \multicolumn{2}{|c|}{2079} & & \multicolumn{3}{|c|}{34,00} & \multicolumn{3}{|c|}{123,13} & \multicolumn{3}{|c|}{3,65} & \multicolumn{2}{|c|}{2,15} & \\
\hline 3 & $2080 \mathrm{a}$ & $2105 \mathrm{a}$ & 2092 & $34,03 a$ & $34,68 \mathrm{a}$ & 34,35 & 118,85 & 120,27 & 119,56 & $3,52 \mathrm{a}$ & $3,50 \mathrm{a}$ & 3,51 & $2,04 \mathrm{a}$ & $2,03 \mathrm{a}$ & 2,03 \\
\hline 6 & $2051 \mathrm{~b}$ & $2144 a$ & 2097 & $33,25 \mathrm{~b}$ & $35,70 \mathrm{a}$ & 34,47 & 118,67 & 121,23 & 119,95 & $3,60 \mathrm{a}$ & $3,42 \mathrm{a}$ & 3,51 & $2,05 \mathrm{a}$ & $1,98 \mathrm{a}$ & 2,01 \\
\hline 9 & $2014 \mathrm{~b}$ & $2116 a$ & 2065 & $32,28 \mathrm{~b}$ & $34,96 a$ & 33,62 & 116,96 & 114,03 & 115,49 & $3,65 \mathrm{a}$ & $3,29 * \mathrm{~b}$ & 3,47 & $2,08 \mathrm{a}$ & $1,90 * \mathrm{~b}$ & 1,99 \\
\hline 12 & $2126 a$ & $1931 * \mathrm{~b}$ & 2028 & $35,24 \mathrm{a}$ & $30,11 * b$ & 32,67 & 117,90 & 118,37 & 118,13 & $3,38 \mathrm{~b}$ & $3,99 * \mathrm{a}$ & 3,68 & $1,89 * \mathrm{~b}$ & $2,32 \mathrm{a}$ & 2,10 \\
\hline Média & 2068 & 2074 & & 33,70 & 33,86 & & 118,09 & 118,47 & & 3,54 & 3,55 & & 2,01 & 2,06 & \\
\hline Regress & \multicolumn{3}{|c|}{ Quadrática } & \multicolumn{3}{|c|}{ Quadrática } & \multirow{2}{*}{\multicolumn{2}{|c|}{5,66}} & $\mathrm{~ns}$ & \multicolumn{2}{|c|}{ Quadrática } & \multicolumn{4}{|c|}{ Quadrática } \\
\hline $\mathrm{CV}$ & \multicolumn{2}{|c|}{6,33} & & \multicolumn{2}{|c|}{10,21} & & & & & \multicolumn{2}{|c|}{10,60} & \multicolumn{4}{|c|}{10,67} \\
\hline
\end{tabular}

DR: dieta referência; PV70: peso vivo 70 dias; GPD 32-70: ganho de peso diário 32-70 dias; CRD 32-70: consumo de ração diário 32-70 dias; CA 32-70: conversão alimentar 32-70 dias; Custo/kg GP32-70: custo das rações, em reais, para cada quilo de ganho de peso dos 32 aos 70 dias de idade dos animais; CV: coeficiente de variação.

*Diferem da referência pelo teste de Dunnett $(\mathrm{P}<0,05)$.

${ }^{a, b}$ Letras na linha representam o desdobramento da interação pelo teste de Tukey $(P<0,05)$. Ausência de letras indica que não houve interação.

ns= não significativo.

PV 70: $\mathrm{Y}=2221,86-56,1759 \mathrm{GSV}+3,9639 \mathrm{GSV}^{2} ; \mathrm{R}^{2}=0,74$ e $\mathrm{Y}=1932,55+74,7828 \mathrm{GSM}-6,2115 \mathrm{GSM}^{2} ; \mathrm{R}^{2}=0,44$

GPD 32-70: $\mathrm{Y}=37,7517-1,4783 \mathrm{GSV}+0,1043 \mathrm{GSV}^{2} ; \mathrm{R}^{2}=0,74$ e $\mathrm{Y}=30,1383+1,9680 \mathrm{GSM}-0,1635 \mathrm{GSM}^{2} ; \mathrm{R}^{2}=0,44$.

CA 32-70: $\mathrm{Y}=3,1930+0,1334 \mathrm{GSV}-0,0097 \mathrm{GSV}^{2} ; \mathrm{R}^{2}=0,70$ e $\mathrm{Y}=4,1959-0,2837 \mathrm{GSM}+0,0219 \mathrm{GSM}^{2} ; \mathrm{R}^{2}=0,54$.

Custo/kg GP 32-70: $\mathrm{Y}=1,8760+0,0680 \mathrm{GSV}-0,0055 \mathrm{GSV}^{2} ; \mathrm{R}^{2}=0,44$ e $\mathrm{Y}=2,4346-0,1664 \mathrm{GSM}+0,0129 \mathrm{GSM}^{2} ; \mathrm{R}^{2}=0,54$.

Com exceção do consumo de ração, todas as variáveis apresentaram comportamento quadrático para ambas as glicerinas. Para a glicerina semipurificada vegetal, foi verificado ponto de mínima de $7,07 \%$ para peso vivo e ganho de peso; e para a conversão alimentar e custo por $\mathrm{kg}$ de peso vivo ganho, pontos de máxima de $6,88 \%$ e $6,18 \%$, respectivamente. Para a semipurificada mista, o ponto de máxima para peso vivo e ganho de peso foi de $6,02 \%$; e para a conversão alimentar e custo por $\mathrm{kg}$ de peso vivo ganho, os pontos de mínima foram de $6,48 \%$ e $6,45 \%$, respectivamente.

O peso vivo e o ganho de peso diário dos animais alimentados com $12 \%$ de inclusão de glicerina semipurificada mista na dieta foram inferiores aos observados nos animais que receberam a ração referência, pelo teste de Dunnett $(\mathrm{P}<0,05)$, assim como a conversão alimentar foi pior com $12 \%$ de inclusão, refletindo os mesmos resultados encontrados para a fase dos 32 aos 50 dias de idade. Desse modo, infere-se novamente que o menor aporte energético metabólico em função da menor porcentagem de ácidos graxos e glicerol apresentada pela glicerina mista pode ter sido responsável por afetar o desempenho. Foi verificada melhor conversão alimentar para os animais que consumiram a dieta com $9 \%$ de inclusão do coproduto; consequentemente, o custo por $\mathrm{kg}$ de peso vivo ganho também foi melhor $(\mathrm{P}<0,05)$ em comparação à dieta referência.

Para a glicerina semipurificada vegetal, a maior viabilidade econômica em relação à referência foi com $12 \%$ de adição da glicerina, apesar de o ganho de peso e o consumo de ração não terem diferido dos animais alimentados com a dieta referência.

Simon et al. (1996), ao avaliarem a inclusão de $5,10,15,20$ e $25 \%$ de glicerina pura na dieta de frangos, concluíram que a inclusão de até $10 \%$ desse produto pode ser utilizado sem afetar o desempenho dos animais. Resultado semelhante foi encontrado por Berenchtein (2010) ao observar que a glicerina semipurificada pode ser utilizada como ingrediente energético das rações de suínos em crescimento e terminação até $9 \%$ de inclusão, sem afetar o desempenho. Já Waldroup (2007), ao conduzir estudos com 
inclusão de 0,5 e $10 \%$ de glicerol, para frangos até os 42 dias de idade, notou que a dieta com inclusão de $10 \%$ do coproduto não fluiu bem nos comedouros, inibindo o consumo de ração, resultando em menor crescimento e pior conversão alimentar. Neste trabalho, a inclusão das glicerinas na dieta não prejudicou a qualidade dos péletes em comparação à referência, apesar de não terem sido avaliados por técnica específica.

No presente trabalho, a glicerina semipurificada mista incluída na dieta na porcentagem de $9 \%$ não influenciou o desempenho dos animais, além de ter melhorado a viabilidade econômica da produção. A dieta com a maior inclusão de glicerina mista, no entanto, propiciou os piores resultados de desempenho dos animais durante todo o período experimental. Já a semipurificada vegetal pode ser incluída até a porcentagem máxima estudada, pois proporcionou desempenho semelhante à dieta referência, inclusive a um custo de produção menor, provavelmente em função da maior quantidade de glicerol e ácidos graxos em relação à semipurificada mista.

Com relação às variáveis apresentadas na Tab. 6, foi detectada interação tipo de glicerina versus porcentagem de inclusão apenas para o peso de carcaça. Essa variável apresentou comportamento quadrático para ambas as glicerinas, com ponto de mínima de 7,26\% para a glicerina vegetal e ponto de máxima de $6,20 \%$ para a mista. Pelo teste de Dunnet $(\mathrm{P}<0,05)$, a dieta com $6 \%$ de inclusão de glicerina vegetal e a dieta com $12 \%$ de inclusão de glicerina mista diferiram negativamente da dieta referência. Houve aumento linear no rendimento de rins para os animais alimentados com porcentagens crescentes de ambas as glicerinas. Aplicando-se o teste de Dunnett $(\mathrm{P}<0,05)$, os animais alimentados com as inclusões de 6, 9 e $12 \%$ de glicerina semipurificada vegetal apresentaram valores superiores para rendimento de rins em relação àqueles da dieta referência e, para a semipurificada mista, os maiores valores foram observados nas inclusões de 9 e $12 \%$. Provavelmente, esse aumento está relacionado com a grande quantidade de glicerol presente nos coprodutos, saturando a enzima glicerol quinase no fígado, responsável pela metabolização do glicerol a glicerol-3-fosfato e aos intermediários da glicólise, dihidroxiacetona fosfato e gliceraldeído-3-fosfato (Lin et al., 1976). Assim, o glicerol que não é metabolizado vai para os rins, onde também existe a enzima. Os rins, na tentativa de metabolizarem o excesso de glicerol vindo do fígado, aumentam a retenção de líquidos e, consequentemente, seu peso. Não houve efeito da inclusão de glicerina $(P>0,05)$ para rendimento de fígado e coração.

Tabela 6. Médias estimadas do rendimento das vísceras de coelhos alimentados, dos 32 aos 70 dias de idade, com dietas contendo glicerina semipurificada vegetal (GSV) e mista (GSM)

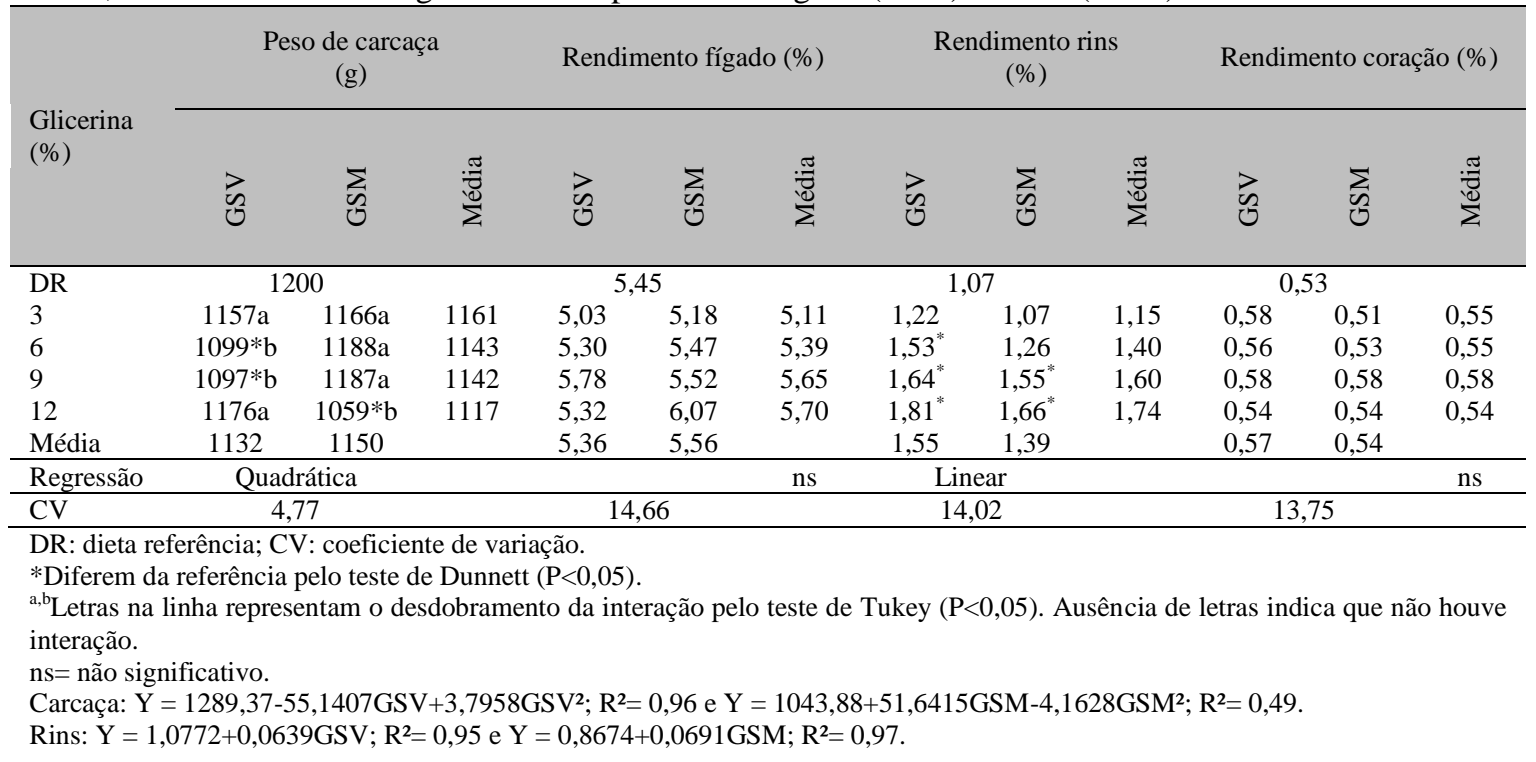




\section{CONCLUSÕES}

As glicerinas semipurificadas vegetal e mista apresentaram energia digestível de 4.048 e $3.697 \mathrm{kcal} / \mathrm{kg} \mathrm{MS}$, respectivamente, mostrando serem fontes altamente energéticas. A glicerina semipurificada vegetal pode ser incluída em até $12 \%$ da dieta, e a semipurificada mista, em até $9 \%$, sem afetarem o desempenho e peso de carcaça dos animais, além de reduzirem o custo de produção.

\section{REFERÊNCIAS}

ADEOLA, O.; ILELEJI, K.E. Comparison of two diet types in the determination of metabolizable energy content of corn distillers dried grains with soluble for broiler chickens by the regression method. Poult. Sci., v.88, p.579585, 2009.

BELLAVER, C.; FIALHO, E.T.; PROTAS, J.F.S. et al. Radícula de malte na alimentação de suínos em crescimento e terminação. Pesq. Agropec. Bras., v.20, p.969-974, 1985.

BERENCHTEIN, B.; COSTA, L.B.; BRAZ, D.B. et al. Utilização de glicerol na dieta de suínos em crescimento e terminação. Rev. Bras. Zootec., v.39, p.1491-1496, 2010.

BLAS, E.; GIDENNE, T. Digestion of starch and sugars. In: DE BLAS, C.; WISEMAN, J. (Ed.). The nutrition of rabbit. Wallingford: CABI, 1998. p.17-38.

BLAS, C.; WISEMAN, J. The nutrition of the rabbit. Wallingford: CABI, 1998. 352p.

GIDENNE, T. Caeco-colic digestion in the growing rabbit: impact of nutritional factors and related disturbances. Livest. Prod. Sci., v.51, p.73-78, 1997.

LAMMERS, P.J.; KERR, B.J.; HONEYMAN, M.S. et al. Nitrogen-corrected apparent metabolizable energy value of crude glycerol for laying hens. Poult. Sci., v.87, p.104-107, 2008.

LIN, M.H.; ROMSOS, D.R.; LEVEILLE, G.A. Effect of glycerol on lipogenic enzyme activities and on fatty acids synthesis in the rat and chicken. J. Nutr., v.106, p.1668-1677, 1976.
MATTERSON, L.D.; POTTER, L.M.; STUTZ, M.W. et al. The metabolizable energy of feed ingredients for chickens. Storrs: The University of Connecticut, Agricultural Experiment Station, 1965. 11p. (Research Report, 7).

OOI, T.L.; YONG, K.C.; HAZIMAH, A.H. et al. Glycerol residue - a rich source of glycerol and medium chain fatty acids. J. Oleo Sci., v.53, p.29-33, 2004.

PEREZ, J.M.; LEBAS, F.; GIDENNE, T. et al. European reference method for in vivo determination of diet digestibility in rabbits. World Rabbit Sci., v.3, p.41-43, 1995.

ROSTAGNO, H.S.; ALBINO, L.F.T; DONZELE, J.L. et al. Tabelas brasileiras para aves e suínos: composição de alimentos $\mathrm{e}$ exigências nutricionais. 1.ed. Viçosa: UFV, 2005.

SILVA, D.J.; QUEIROZ, A.C. Análise de alimentos: métodos químicos e biológicos. 3.ed. Viçosa: UFV, 2002.

SIMON, A.; BERGNER, H.; SCHWABE, M. Glycerol as a feed ingredient for broiler chickens. Arch. Anim. Nutr., v.49, p.103-112, 1996.

SISTEMA para análises estatísticas - SAEG, versão 9.1. Viçosa: UFV, 2007.

SÜDEKUM, K.H. Co-products from biodiesel production. In: GARNSWORTHY, P. C.; WISEMAN, J. (Ed.) Recent advances in animal nutrition. Notthingham: Notthingham University Press, 2008. p.210-219.

WALDROUP, P.W. Biofuels and broilers: competitors or cooperators? In: MIDATLANTIC NUTRITION CONFERENCE, 5., 2007, Timonium. Proceedings... Timonium: University of Maryland, 2007. p.25-34

YONG, K.C.; OOI, T.I.; DZULKEFLY, K. et al. Characterization of glycerol residue from a palm kernel oil methyl ester plant. J. Oil Palm Research, n.13, p.39-42, 2001. 